\title{
GENERACIÓN DE MODELO PETROFÍSICO-ESTRATIGRÁFICO DEL CAMPO CHIMIRE, VENEZUELA
}

\author{
Naire M. Castro I. ${ }^{1 *}$ Ignacio J. Mederos V. ${ }^{2}$ \\ * A quien debe dirigirse la correspondencia.
}

\begin{abstract}
RESUMEN
Con el propósito de determinar nuevos modelos petrofísicos-estratigráficos del campo Chimire, ubicado en la Faja Petrolífera del Orinoco en Venezuela, se utilizaron los registros eléctricos y radiactivos de 81 pozos para la elaboración y el análisis de mapas de isopropiedades petrofísicas. Se contó con 3 pozos con descripción de núcleo (Pozo A, Pozo D y Pozo GV117) a partir de los cuales se definieron los topes estructurales de las formaciones: Formación Freites, Formación Oficina (Miembro Morichal Superior, Miembro Morichal Medio y Miembro Morichal Inferior). De las mismas se observaron estructuras que responden a fallamiento tipo flor y a fallamiento normal que presentan saltos de 250 a 500 pies. Posteriormente se determinaron 10 eventos estratigráficos: 5 eventos transgresivos y 5 eventos regresivos, que sirvieron como marcadores litoestratigráficos.
\end{abstract}

Luego de definido el modelo estratigráfico, se generaron mapas de isopropiedades petrofísicas a partir de los parámetros físicos de la roca que se utilizaron para la determinación de tres (3) nuevos prospectos exploratorios, a los cuales se les calculó el petróleo original en sitio (POES) mediante el método estadístico de simulación de Monte Carlo con el fin de determinar la factibilidad de recobro de los mismos.

Palabras Claves: campo Chimire, Petróleo Original en Sitio (POES), Estratigrafía, secuencia estratigráfica, registros de pozo, mapas isopacos y de isopropiedades petrofísicas.

\section{GENERATION OF A PETROPHYSICAL AND STATIGRAPHICAL MODEL OF THE CHIMIRE OIL FIELD, ANZOÁTEGUISTATE, VENEZUELA}

In order to determine new stratigraphic petrophysical models of Chimire field in the Orinoco Oil Belt in Venezuela, electrical and radioactive records of 81 wells for the production and analysis of petrophysical properties maps they were used. It featured three wells with description of core (Well A, Well D and Pozo GV117) from which the structural ceilings formations were defined: Freites Formation, Office Formation (Morichal Top Member, Morichal Middle Member and Morichal Lower Member). The same structures that respond to flower type and normal faulting presenting jumps 250-500 feet were observed. Subsequently, 10 stratigraphic events were determined: five transgressive events and five regressive events, which served as lithostratigraphic markers.

After defined the stratigraphic model, petrophysical maps were generated from the physical parameters of the rock that were used for the determination of three (3) new exploration prospects to which they calculated the original oil in place (POES ) using the statistical method of Monte Carlo simulation in order to determine the feasibility of recovery thereof.

Keywords: Chimire field, Original Oil In Place (POES), stratigraphy, stratigraphic sequence, well logs, isopach maps and maps of petrophysical properties.

1. Escuela de Geología, Minas y Geofísica. Universidad Central de Venezuela, Caracas, Venezuela. Email: Naire.M.Castro@gmail.com

2. Escuela de Geología, Minas y Geofísica. Universidad Central de Venezuela, Caracas, Venezuela. Email: imederos30@gmail.com 


\section{INTRODUCCIÓN}

Una caracterización petrofísica-estratigráfica, requiere integrar diversos aspectos: propiedades físicas de las rocas y fluidos presentes en la misma (porosidad, permeabilidad, saturación de fluido, entre otros) e interpretación de distintos registros de pozos, con el objetivo de relacionar los eventos estratigráficos (regresiones, transgresiones) ocurridos en un pasado geológico, que conllevaron a la disposición actual de los depósitos sedimentarios presentes en el subsuelo.

De acuerdo a la integración de estos aspectos, se llevó a cabo la presente investigación, la cual se realizó en la cuenca de mayores recursos petrolíferos de América del Sur: la Cuenca Oriental de Venezuela, en donde se encuentra la Faja Petrolífera del Orinoco (González De Juana et al. 1980). Debido al interés económico probado de la Cuenca Oriental de Venezuela, existe una gran variedad de estudios e investigaciones geológicogeofísico y de yacimiento, realizadas en los distintos campos petroleros existentes en el área. No obstante, existen aún campos en los cuales no se tiene suficiente información o definición estratigráfica, como es el caso del campo Chimire, perteneciente al Área Mayor de Oficina, estado Anzoátegui, campo en el cual se desarrolló el presente estudio (Figura 1).

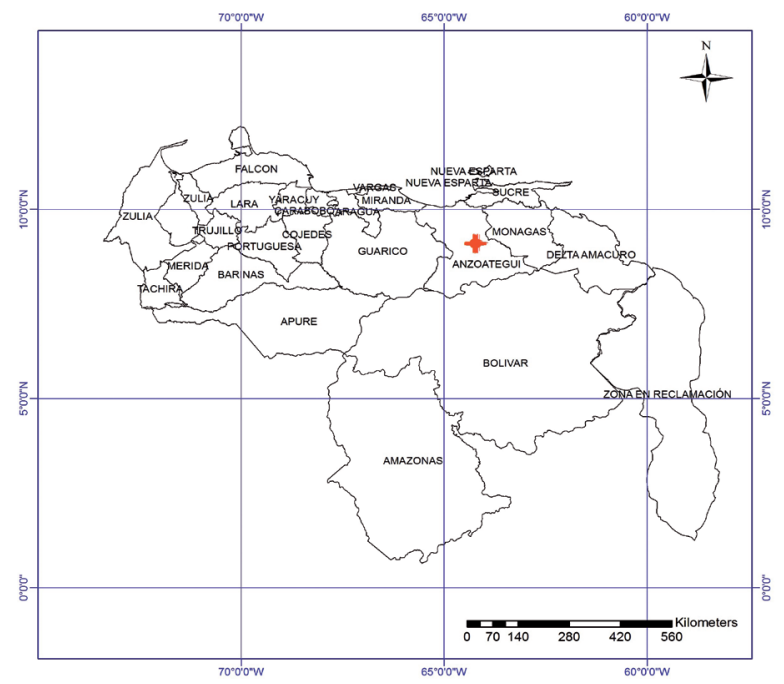

Figura 1. Ubicación del área de estudio: campo Chimire, estado Anzoátegui (Castro, 2015).

El campo Chimire, tiene una extensión de $240 \mathrm{~km}^{2}$. El mismo fue descubierto por la Socony-Vacuum Oil Company de Venezuela en 1948 y para el 1 de enero de 1951, alrededor de 104 pozos ya se habían perforado, resultando en una producción de más de 21.789 .000 barriles y una producción diaria aproximada de 55.265 barriles. (Moore \& Shields, 1952).

En el año 1997, se llevó a cabo el levantamiento sísmico Chimire 3D, con una extensión de $219 \mathrm{~km}^{2}$ y contó con un total de 216 pozos con su respectiva data (marcadores/registros) esparcidos por toda el área de interés y 71 pozos con data petrofísica. Este proyecto, sirvió de inicio para posteriores estudios geofísicos de interpretación sísmica (Quijada \& Beltrán, 2001).

Otro autores: Carrero (2013), Fajardo \& Izarra (2003), Larrañaga (2000) y Velásquez (2003), realizaron estudios similares en el campo, a fin de conocer a profundidad los yacimientos y zonas de interés petrolíferos para aportar nuevas interpretaciones y, en consecuencia, optimizar la explotación de hidrocarburo en el área.

Es así, como en la presente investigación, se propone un modelo petrofísico en la zona, a partir de la correlación lateral de los topes estructurales de las formaciones: Formación Freites, Formación Oficina (Miembro Morichal Superior, Miembro Morichal Medio y Miembro Morichal Inferior), y un modelo estratigráfico a partir de la correlación de eventos transgresivos y regresivos observados en los registros de rayos gamma.

Se contó con registros petrofísicos de 81 pozos distribuidos en el campo Chimire, el cual representa $219 \mathrm{~km}^{2}$ aproximadamente y está delimitado por las siguientes coordenadas:

$$
\begin{gathered}
\text { A }(349055,1011000) \\
\text { B }(371700,101100) \\
\text { C }(371700,1001001) \\
\text { D }(349055,1001001)
\end{gathered}
$$

En este sentido, se generaron e interpretaron mapas de isopropiedades petrofísicas con la finalidad de proponer prospectos petroleros exploratorios, definiendo el interés económico de los mismos a partir de un análisis estadístico, utilizando el método de simulación de Monte Carlo, que permitió el cálculo del petróleo original en sitio (POES) de cada uno de los prospectos propuestos.

\section{MARCO GEOLÓGICO- ESTRATIGRÁFICO}

La Cuenca Oriental de Venezuela está ubicada en la región centro-este del país y se define como una gran depresión topográfica y estructural cuyos límites son: al sur con el borde septentrional del Cratón de Guayana, al 
norte con el cinturón móvil de las Serranías del Interior Central y Oriental, y al oeste limita con el levantamiento de El Baúl (Fajardo \& Izarra, 2003).

La longitud aproximada de la Cuenca Oriental de Venezuela es de $800 \mathrm{~km}$ en sentido oeste-este con una anchura promedio de $200 \mathrm{~km}$ de norte a sur. La cuenca actual es asimétrica, debido a esto el flanco sur está ligeramente inclinado hacia el norte mientras que el flanco norte está más tectonizado y posee mayores buzamientos; en consecuencia, se ha obtenido producción de petróleo de ambos flancos de la cuenca pero bajo condiciones estratigráficas y estructurales diferentes (González De Juana et al. 1980).

\section{GeOLOGíA LOCAL}

La producción de hidrocarburos en el Campo Chimire, principalmente es a partir de las arenas de la Formación Oficina por excelencia, a la cual le sigue en importancia la Formación Merecure $\mathrm{y}$, por último, las areniscas basales de la Formación Freites. La Formación Oficina está relacionada a una combinación de trampas estratigráficas y fallas que ofrecen una barrera a la migración de hidrocarburo, esto debido al sistema de fallas este-oeste (Moore \& Shields, 1952).

Por lo mencionado anteriormente, a continuación se describen las principales formaciones geológicas a interpretar en la presente investigación, asociadas al campo en estudio, perteneciente al Área Mayor de Oficina.

- Formación Oficina (Mioceno Temprano Medio): se ve representada en el subsuelo de los estados Anzoátegui y Monagas, formando parte de las unidades de la cuenca oriental de Venezuela. Audemaret al. (1985) describen la Formación Oficina de la Faja Petrolífera del Orinoco, dividiéndola en tres unidades: la Unidad I (Miembro Morichal del área de Cerro Negro; Formación Oficina Inferior en Zuata) caracterizada por areniscas masivas progradantes y la intercalación de lutitas y areniscas transgresivas; Unidad II (Miembro Yabo del área de Cerro Negro;
Formación Oficina Media en Zuata) representada por una secuencia lutítica con intercalaciones ocasionales de areniscas y limolitas; y la Unidad III (miembros Jobo y Pilón del área de Cerro Negro; Formación Oficina Superior de Zuata) que es una secuencia predominantemente arenosa.

- Formación Freites (Mioceno Medio a Mioceno Tardío basal): Representativo en el estado Anzoátegui, la Formación Freites se extiende en el subsuelo a través de todo el flanco sur de la subcuenca de Maturín casi hasta el río Orinoco. Para esta unidad, se describe una litología de lutitas en tonos verdosos, siguiendo con areniscas en el tope y en la base de la formación (González de Juana et al., 1980).

\section{ASPECTOS ESTRUCTURALES DEL ÁREA MAYOR DE OFICINA}

La investigación se realizó, gracias a registros de pozos provenientes del Área Mayor de Oficina, zona que pertenece al flanco sur de la Cuenca Oriental de Venezuela. Dicha cuenca, presenta una estructura tipo homoclinal con suave buzamiento hacia el norte y es cruzada por tres sistemas de fallas normales. Se mencionan por su extensión regional, las fallas de GuaraLeona, las cuales atraviesan los campos petrolíferos Guara Este y Guara Central, así como la Falla del Merey, ambas con buzamiento hacia el norte. Sigue en importancia un sistema con rumbo NW-SE, más notable en la región suroeste del Área Mayor de Oficina donde ocurre la falla Matapalo con una traza aproximadamente de $30 \mathrm{~km}$ de longitud (Fajardo \& Izarra, 2003).

También es importante resaltar, la falla de Tascabaña de orientación EO, la cual fue la principal falla observada para los resultados obtenidos en el proyecto sísmico Chimire 3D. Quijada y Beltrán (2001), elaboraron una sección transversal noroeste-sureste, en donde se observan las variaciones en las estructuras tectónicas asociadas a la cuenca, mostrando una estructura tipo flor positiva, característica de la zona del Área Mayor de Oficina (Figura 2). 


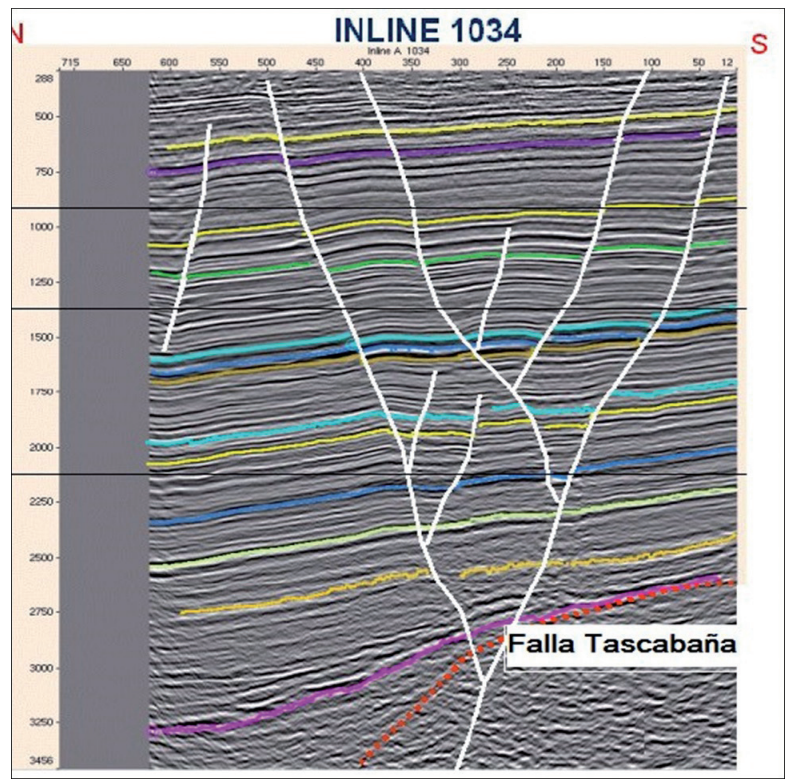

Figura 2. Corte Oblicuo (Proyecto sísmico Chimire 3D 1997), estructura tipo flor positiva observándose la falla Tascabaña (Quijada \& Beltrán, 2001).

\section{ASPECTOS ESTRATIGRÁFICOS}

Un sistema fluvio-deltaico de una plataforma de bajo ángulo de $2^{\circ}$ a $4^{\circ}$, representa a la zona en estudio, en donde, según antecedentes geológicos previos (Albertini \& Cadena (2014), Chramcow (2008), Sandia (2004)), se plantea un tipo de secuencia estratigráfica tipo 2 (exposición parcial de la plataforma) debido a la baja angularidad de la misma. Este aspecto, se corrobora a partir de la correlación litoestratigráfica, realizada mediante los registros de rayos gamma (GR), en donde se observa la continuidad lateral de dichos eventos, al delimitar regresiones y/o transgresiones, de topes litoestratigráficos. En la Figura 3, se ilustran las típicas respuestas de registros de pozos para eventos regresivos $\mathrm{y}$ transgresivos.

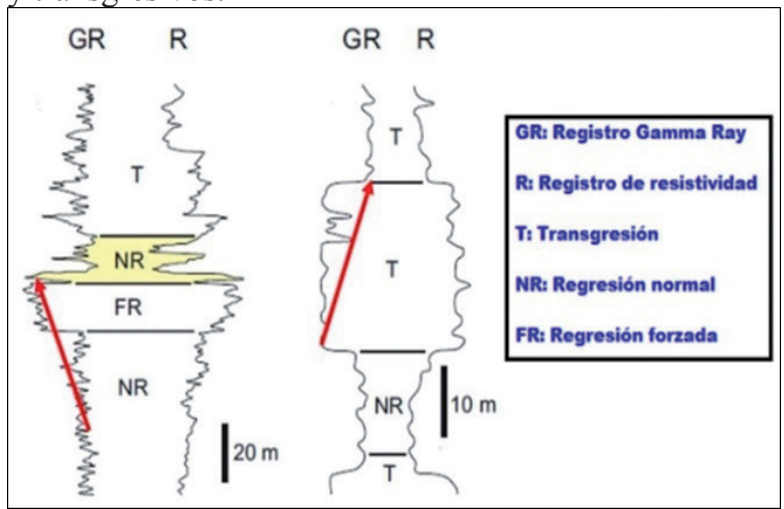

Figura 3. Respuesta de registros Gamma Ray de acuerdo a eventos estratigráficos. Tomado de Catuneanuet al. (2011). Modificado por Castro (2015).
Dichos limites litoestratigráficos permiten la identificación de sistemas sedimentarios, los cuales son fundamentales para entender el pasado geológico de la zona en estudio, ya que permiten relacionar aspectos relevantes (granulometría, energía y dirección de transporte, entre otros) de las secuencias sedimentarias que componen el yacimiento.

\section{MÉTODO}

El esquema de trabajo se muestra en la Figura 4, en donde como primera etapa, se buscó recopilar la mayor información previa del campo Chimire. Inicialmente se cargaron 164 pozos, pero de acuerdo a un inventario realizado para los tipos de registros que se tenían por cada pozo, se trabajó con 81 pozos en total.

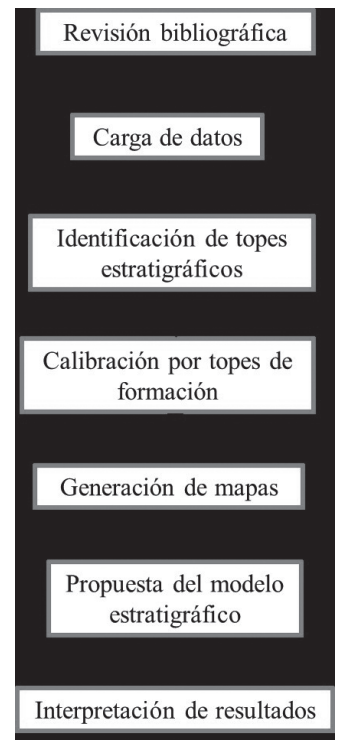

Figura 4. Secuencia general de trabajo en el desarrollo del proyecto.

Una etapa previa a la delimitación de los eventos estratigráficos y estructurales, fue la de establecer una relación entre los horizontes dados en tiempo con los topes formacionales conocidos de los pozos en profundidad; la misma se realizó mediante el cálculo de las profundidades de los topes de Formación de acuerdo a información precedente de investigaciones anteriores: Velásquez (2003) y Fajardo \& Izarra (2003), obteniendo la estimación de las velocidades promedios de las formaciones con las cuales se trabajó. Se utilizó la ecuación 1 para calcular la profundidad de cada horizonte.

$$
\mathrm{P}=\left(\text { Vprom }^{*} \mathrm{~T}\right) / 2
$$

Donde,

Vprom $=$ Velocidad promedio del horizonte $(\mathrm{ft} / \mathrm{ms})$ 
$\mathrm{T}=$ tiempo doble $(\mathrm{ms})$

$\mathrm{P}=$ Profundidad del horizonte escogido $(\mathrm{ft})$

Esto se realizó gracias a 3 pozos guía (Figura 5) en donde se contaba con la información en profundidad, de las principales formaciones geológicas del área, obtenidos de trabajos previos (Fajardo \& Izarra (2003), Quijada \& Beltrán, 2001).

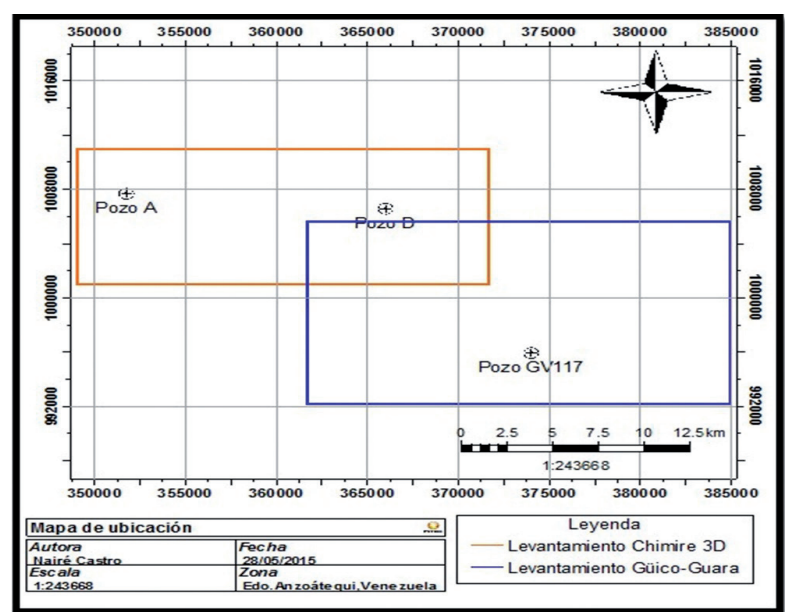

Figura 5. Distribución de los pozos núcleo utilizados para la correlación.

\section{METOdOLOGÍA ESTRATIGRÁFICA- ESTRUCTURAL}

Se delimitaron los topes estratigráficos para 81 pozos que contenían registros de rayos gamma (Figura 6); la escogencia de dichos topes, se realizó de acuerdo a los máximos en arena y mínimos en lutitas observados en los registros de rayos gamma de los pozos, los cuales corresponden a eventos estratigráficos progradantes $\mathrm{y}$ retrogradantes, respectivamente.

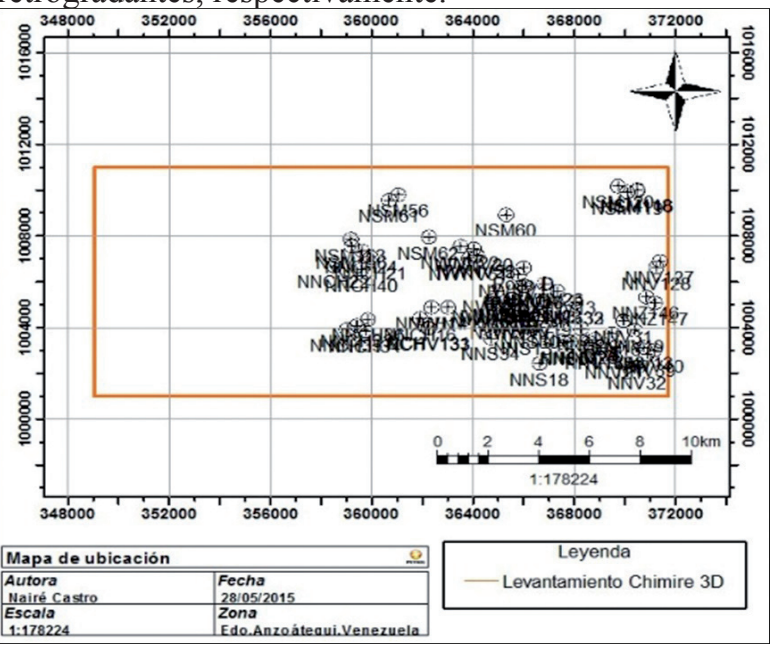

Figura 6. Mapa de ubicación de los pozos utilizados para las correlaciones (Castro,2015).
Con respecto a los topes estructurales definidos, se identificaron mediante la combinación de los horizontes convertidos de tiempo a profundidad (ecuación 1) y los topes estructurales en profundidad de los pozos guía (núcleos), para establecer la posterior correlación lateral de los topes formacionales.

De acuerdo a esto, se consiguieron establecer tres zonas delimitadas entre los topes estructurales: Zona 1 (Tope Freites a Formación Oficina, Miembro Morichal Superior), Zona 2 (Formación Oficina: Miembro Morichal Superior a Miembro Morichal Medio) y Zona 3 (Formación Oficina: Miembro Morichal Medio a Miembro Morichal Inferior).

\section{CORRELACIÓN POR TOPES ESTRATIGRÁFICOS-ESTRUCTURALES}

En esta etapa de la investigación, los eventos estratigráficos y topes estructurales delimitados anteriormente, se utilizaron para establecer correlaciones laterales de cada uno de los pozos, migrando así la información en profundidad, con el objetivo de estudiar la continuidad lateral de los horizontes. Un ejemplo de esto, se muestra en la Figura 7.

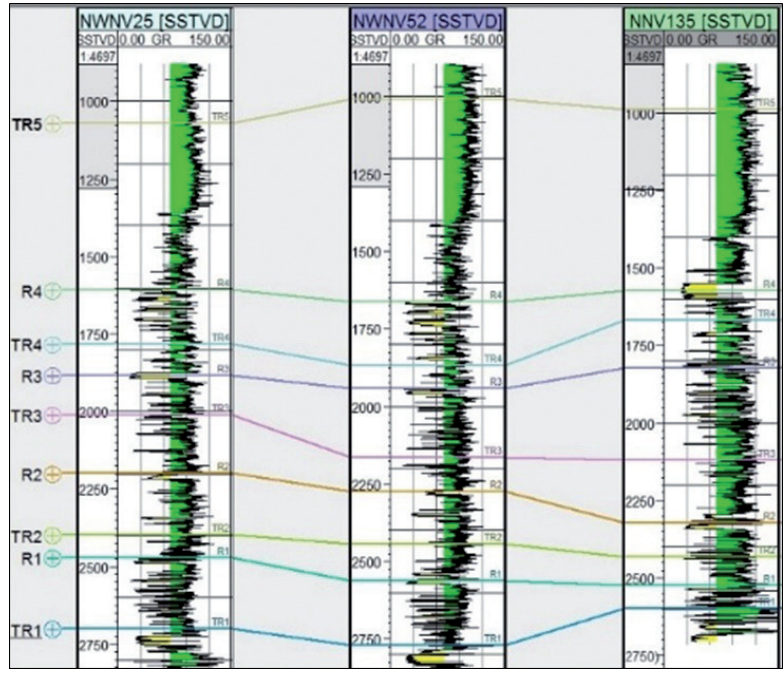

Figura 7. Ejemplo de continuidad lateral para topes estratigráficos en 3 pozos, siendo TRi: evento transgresivo i y Ri: evento regresivo i (Castro,2015).

\section{METODOLOgÍA PETROFÍSICA}

Se crearon los atributos continuos de Saturación de Agua (Sw), Volumen de arcilla (Vsh), Arena Neta $(\mathrm{AN})$, Arena Neta Petrolífera (ANP), Porosidad ( $\Phi)$ y Saturación de petróleo (So), en base a las condiciones mencionadas en la tabla 1. 
Para cada propiedad, se creó un mapa de isopropiedades petrofísica.

Tabla 1. Tabla resumen de los parámetros utilizados para la generación de los 4 registros.

\begin{tabular}{|c|c|c|c|}
\hline $\begin{array}{l}\text { Tipo de } \\
\text { Registro }\end{array}$ & $\begin{array}{l}\text { Creado a } \\
\text { partir del } \\
\text { registro }\end{array}$ & $\begin{array}{c}\mathrm{N}^{\circ} \text { de } \\
\text { pozos } \\
\text { utilizados }\end{array}$ & $\begin{array}{c}\text { Condición } \\
\text { utilizada }\end{array}$ \\
\hline $\begin{array}{l}\text { Saturación } \\
\text { de } \\
\text { Petróleo } \\
\text { (So) }\end{array}$ & $\begin{array}{c}\text { Saturación de } \\
\text { Agua (Sw) }\end{array}$ & 30 & $\mathrm{So}=1-\mathrm{Sw}$ \\
\hline $\begin{array}{c}\text { Arena } \\
\text { Neta (AN) }\end{array}$ & $\begin{array}{c}\text { Gamma Ray } \\
\text { (GR) }\end{array}$ & 70 & $\mathrm{GR}<=70$ \\
\hline $\begin{array}{l}\text { Arena } \\
\text { Neta } \\
\text { Petrolífera } \\
\text { (ANP) }\end{array}$ & $\begin{array}{c}\text { Gamma } \\
\text { Ray (GR), } \\
\text { Resistividad } \\
\text { (ILD) }\end{array}$ & 67 & $\begin{array}{c}\mathrm{GR}<=70 \& \\
\mathrm{ILD}>=100\end{array}$ \\
\hline $\begin{array}{c}\text { Volumen } \\
\text { de arcilla } \\
\text { (Vsh) }\end{array}$ & $\begin{array}{c}\text { Gamma Ray } \\
\text { (GR) }\end{array}$ & 70 & $\begin{array}{c}\text { Vsh }=(\text { GR }- \\
\text { Grmax }) /(\text { Grmax } \\
\text { - GRmín) }\end{array}$ \\
\hline
\end{tabular}

En este sentido, se crearon un total de 18 mapas, 6 por cada zona. En la Figura 5 el recuadro naranja, representa el área que abarcaron los mapas creados.

Por otra parte, se crearon dos perfiles, con la finalidad de observar la continuidad lateral y comportamientos estructurales y corroborar el ambiente depositacional en base a los topes deFormacióny a los eventos estratigráficos delimitados previamente, respectivamente. La selección de la orientación de los perfiles, se basó en observar las variaciones en las respuestas laterales de los registros rayos gamma y por ende en los cambios en profundidad para los topes de Formación y los topes de eventos estratigráficos; es por esta razón, que se realizó un perfil perpendicular a la dirección de las principales fallas de la zona (Perfil AB N50O, Figura 8), y otro paralelo a estas estructuras geológicas (Perfil CD N60E, Figura 9).

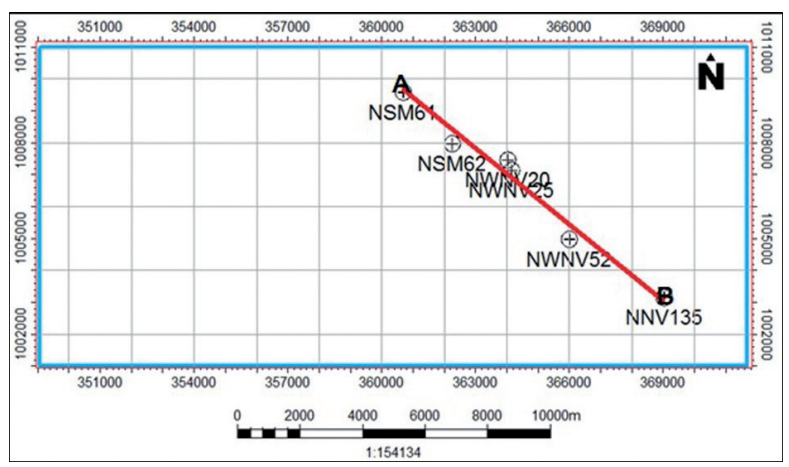

Figura 8. Ubicación del perfil AB N50O.

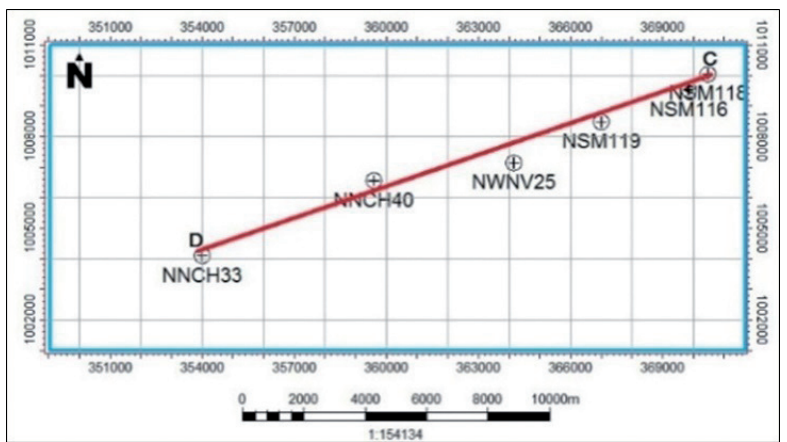

Figura 9. Ubicación del perfil CD N60E.

\section{METOdOLOgÍA de YACIMIENTO PARA EL CÁlCULO DE POES}

En base a las características litológicas del subsuelo, se presentan zonas con posibilidades de albergar hidrocarburos en ella, debido a la presencia de trampas estructurales definidas como la estructura en flor positiva y los controles estratigráficos creados por el sistema dominante del ambiente fluvio-deltaico, que permiten la presencia de altas porosidades y permeabilidades. Estas características, permiten la consolidación de ciertos sectores del yacimiento, con arenas lenticulares como rocas reservorios.

Existen varios métodos para el cálculo de reservas de petróleo, sin embargo, la técnica utilizada en esta investigación fue a través de un Balance Volumétrico, lo que permitió cuantificar el volumen neto de petróleo existente en el yacimiento, en base al volumen total del mismo (Santaniello, 2003).

De acuerdo a Martínez (2007), este método permite la estimación estadística del petróleo original en sitio (POES) a partir de la determinación del volumen de roca que conforma el yacimiento, la capacidad de almacenamiento y la fracción de hidrocarburos presentes en los poros de dicha roca. De tal manera se procedió a utilizar la ecuación para la determinación de Petróleo Original en Sitio (POES) siguiente:

$$
\text { POES }=7758^{*} A^{*} h^{*} \Phi^{*} \text { So*1/Boi }
$$

Donde,

POES $=$ Petróleo Original en Sitio $(\mathrm{BN})$

$\mathrm{A}=$ Área (acres)

$\mathrm{H}=$ espesor (pies)

$\Phi=$ Porosidad (\%)

$\mathrm{So}=$ Saturación de Petróleo $(1-\mathrm{Sw})$

$\mathrm{Sw}=$ Saturación de agua

Boi $=$ Factor volumétrico del petróleo $(\mathrm{BY} / \mathrm{BN})$ 
Basado en la ecuación anterior, se utilizó el Método de Monte Carlo el cual consiste en realizar "N" simulaciones, variando parámetros de la ecuación (ecuación 2), en base a las características propias de cada prospecto definido y a los rangos de variación, en profundidad, de cada parámetro en los pozos cercanos a cada prospecto (Santaniello, 2003).

Se calculó el petróleo original en sitio (POES) de los prospectos definidos mediante la interpretación previa de los valores petrofísicos observados en los distintos mapas de isopropiedades generados en las etapas anteriores. Esto se logró, mediante un análisis estadístico de la zona, haciendo uso de una hoja de cálculo para una simulación del método de Monte Carlo utilizando 10000 iteraciones en el software "Oracle CrystalBall".

El volumen se calculó, mediante la ecuación 3, en donde se utilizó el valor aproximado del polígono delimitado para los prospectos, multiplicado por el espesor de Arena Neta Petrolífera dado en el mapa de esta propiedad.

$$
\mathrm{V}=\mathrm{A}^{*} \mathrm{H}
$$

Donde, $\mathrm{V}=$ volumen el prospecto propuesto

$\mathrm{A}=$ área aproximada del prospecto propuesto

$\mathrm{H}=$ espesor de Arena Neta Petrolífera

Los valores de porosidad y saturación de agua se hallaron, utilizando los valores de los registros petrofísicos de los pozos cercanos al área del prospecto.

Es por esto y por distribuciones previas (Villalba (2011), Carrero (2013)) utilizadas en el campo, que para los valores de porosidad y saturación de agua, se trabajó con una distribución logarítmica, mientras que para el espesor, se utilizó una distribución triangular. Los datos de área y factor volumétrico, fueron constantes durante todo el proceso por ser valores constantes.

En la tabla 2, se muestra un resumen de los rangos utilizados en el análisis estadístico realizado para la simulación Monte Carlo. El valor del factor volumétrico (Boi) fue de 1,1143 BN/BY, valor que corresponde a las características oficiales del campo Chimire (Carrero, 2013).

Tabla 2. Tabla resumen de los parámetros utilizados para el cálculo de POES.

\begin{tabular}{ccccc} 
Zona & Área (acres) & Espesor (pies) & Porosidad & Sw \\
\hline 1 & 355,67 & 100' a $200^{\prime}$ & 0,0013 a 0,3 & 0,23 a 0,6 \\
\hline 2 & 355,67 & $4^{\prime}$ ' a $9^{\prime}$ & 0,03 a 0,06 & 0,52 a 0,8 \\
\hline 3 & 355,67 & $2,5^{\prime}$ a 7 & 0,02 a 0,04 & 0,7 a 0,9 \\
\hline
\end{tabular}

\section{RESULTADOS Y DISCUSIÓN}

Para caracterizar petrofísicamente las arenas del campo, se evaluaron los pozos que se encontraban dentro de los yacimientos en estudio, para un total de 81 pozos, los cuales tenían registros radioactivos de densidad, rayos gamma, neutrón y registros eléctricos de resistividad profunda. Con esta muestra, se realizó el análisis basándose en los métodos de Serra (1985) y Serra \& Baldwin (1980). Se crearon los mapas de isopropiedades, resaltando características que indican la capacidad de almacenamiento de hidrocarburo en zonas del campo, a fin de observar las variaciones en el intervalo definido, para indicar el área con mejores propiedades petrofísicas $\mathrm{y}$ así proponer prospectos exploratorios.

Los mapas de Arena Neta Petrolífera (ANP), se obtuvieron utilizando un punto de corte (cut off) del registro de rayo gamma, al 50\% del rango de valores. Posteriormente a este procedimiento en todos los pozos, se obtuvo una distribución continua de esta propiedad, con la cual se logró tener una apreciación aproximada de la geometría de los cuerpos de arenas limpias existentes en el área y un punto de corte de registros eléctricos mayores a $100 \mathrm{ohm}$ x m para garantizar arenas con petróleo. Ambos puntos de corte se establecieron de acuerdo a las condiciones litológicas del campo. De dicho análisis se obtuvieron espesores de arena neta petrolífera de 10 pies a 250 pies como máximo valor de espesor. En la Formación Oficina Miembro Morichal Superior es donde se encontraron los mayores espesores de arena neta entre 120 a 250 pies.

En el mapa de esta propiedad, para la zona 1 (Figura 10 ), se evidencia un aumento de espesores hacia la zona centro y noreste del área abarcada por el levantamiento sísmico Chimire 3D. En esta zona, se ubican los mayores valores de espesores de Arena Neta Petrolífera, entre 120 a 250 pies de espesor, sugiriendo la ubicación de un posible primer prospecto. Obsérvese la distribución de los espesores en el mapa, en donde los mayores espesores 
quedan representados por el polígono de color rojo, en donde el gradiente de las curvas isópacas es mayor. Las zonas restantes del mapa presentan pocas variaciones, presentando valores menores a 20 pies de espesor.

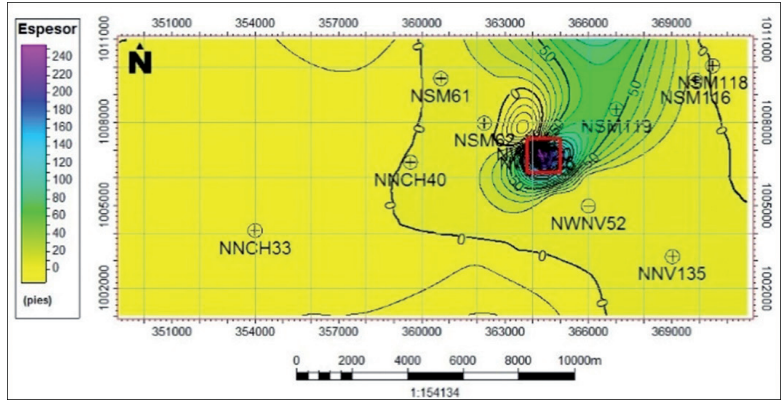

Figura 10. Mapa de Arena Neta Petrolífera (ANP) Zona 1.

Para los mapas de Arena Neta Petrolífera de la Zona 2 (Formación Oficina Miembro Morichal Medio) se observó una disminución considerable en cuanto a los valores de espesores con hidrocarburos, esto, debido a la presencia de arenas más arcillosas en dicho miembro. El mapa de la zona 2 representa valores de espesores de 1 a 6 pies, sin embargo, se evidencia en la zona central, valores máximos de espesores de arena neta petrolífera de 11 pies. Esta zona, se ubica inmediatamente por debajo del prospecto 1, propuesto en el mapa anterior, representado por la Formación Oficina Miembro Morichal Superior, Formación suprayacente a la actual, por lo cual se propone un prospecto 2, delimitado por el polígono rojo (figura 11). Se puede observar que sigue la tendencia en ésta zona en específico, a representar los mejores valores de arena neta petrolífera a lo largo del campo.

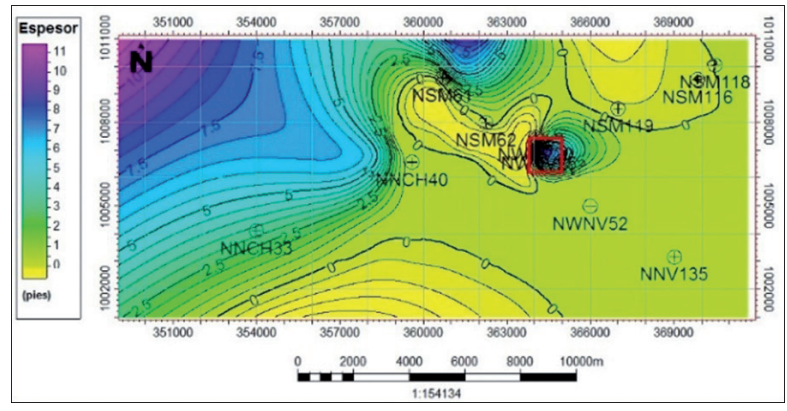

Figura 11. Mapa de Arena Neta Petrolífera (ANP) Zona 2.

Por otra parte, el mapa de Arena Neta Petrolífera de la zona 3 (Formación Oficina Miembro Morichal Inferior), representa los menores valores de espesores de arena neta petrolífera (ANP) en comparación con las demás zonas. Los valores oscilan entre 3 y 10 pies de espesor de ANP. Se señala la zona central-este, en donde se puede considerar un tercer prospecto con valores de espesor de 3 a 6.5 pies (Figura 12).

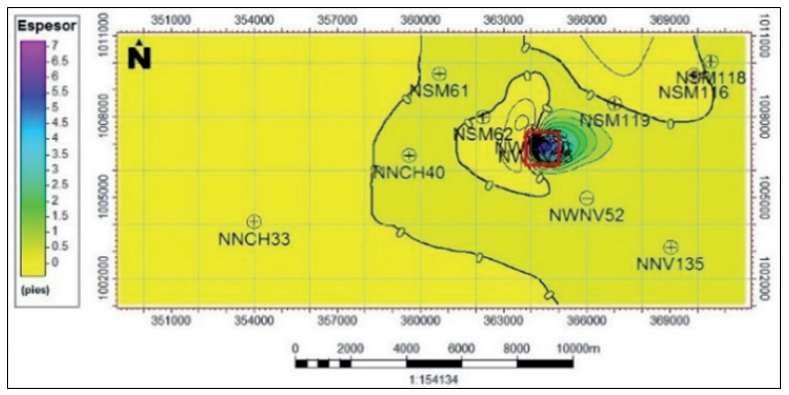

Figura 12. Mapa de Arena Neta Petrolífera(ANP) Zona 3.

Los valores observados en los mapas realizados para los demás atributos (Saturación de Agua ( $\mathrm{Sw}$ ), Volumen de arcilla (Vsh), Arena Neta (AN), Porosidad ( $\Phi)$ y Saturación de petróleo (So)), confirman lo determinado en los mapas de Arena Neta Petrolífera, afianzando la localización de los prospectos propuestos en las zonas estudiadas; se observaron las mejores propiedades de calidad de roca en el yacimiento en estudio, a distinta profundidad, con valores menores a $15 \%$ de volumen de arcilla y porosidades de hasta 35\% (Castro, 2015). La ubicación de los 3 prospectos, se representa por un polígono de color rojo en los mapas señalados anteriormente, entre las coordenadas UTM 364000 y 366000 .

Por otra parte, al construir el perfil AB N50O, el cual se realizó siguiendo la dirección preferencial de las fallas principales del campo, se observó una tendencia marcada de los topes formacionales a profundizar de norte a sur, siendo coherente con la forma de la cuenca y la cercanía al rio Orinoco al Sur que reduce el espacio de depositación de sedimentos. También se puede observar que a partir de los extremos del perfil de correlación, hay una profundización que aumenta hacia el centro del mismo, asemejándose a la estructura en flor debido a fallas normales que originan un graben en la zona (Figura 13).

Además, se pudo determinar en base a los pozos extremos de sur a norte, una somerización al sur con rangos de entre 1950 pies para el tope de la Formación Oficina (Miembro Morichal Superior) y 2050 pies para el tope representado por la Formación Oficina Miembro Morichal Inferior. Mientras que en el extremo norte se observa la profundización de los topes estructurales con 2500 pies aproximadamente para el tope de la Formación Oficina (Miembro Morichal Superior) y 2600 pies para el tope de Formación Oficina (Miembro Morichal Inferior) evidenciando las características estructurales del Área Mayor de Oficina. 


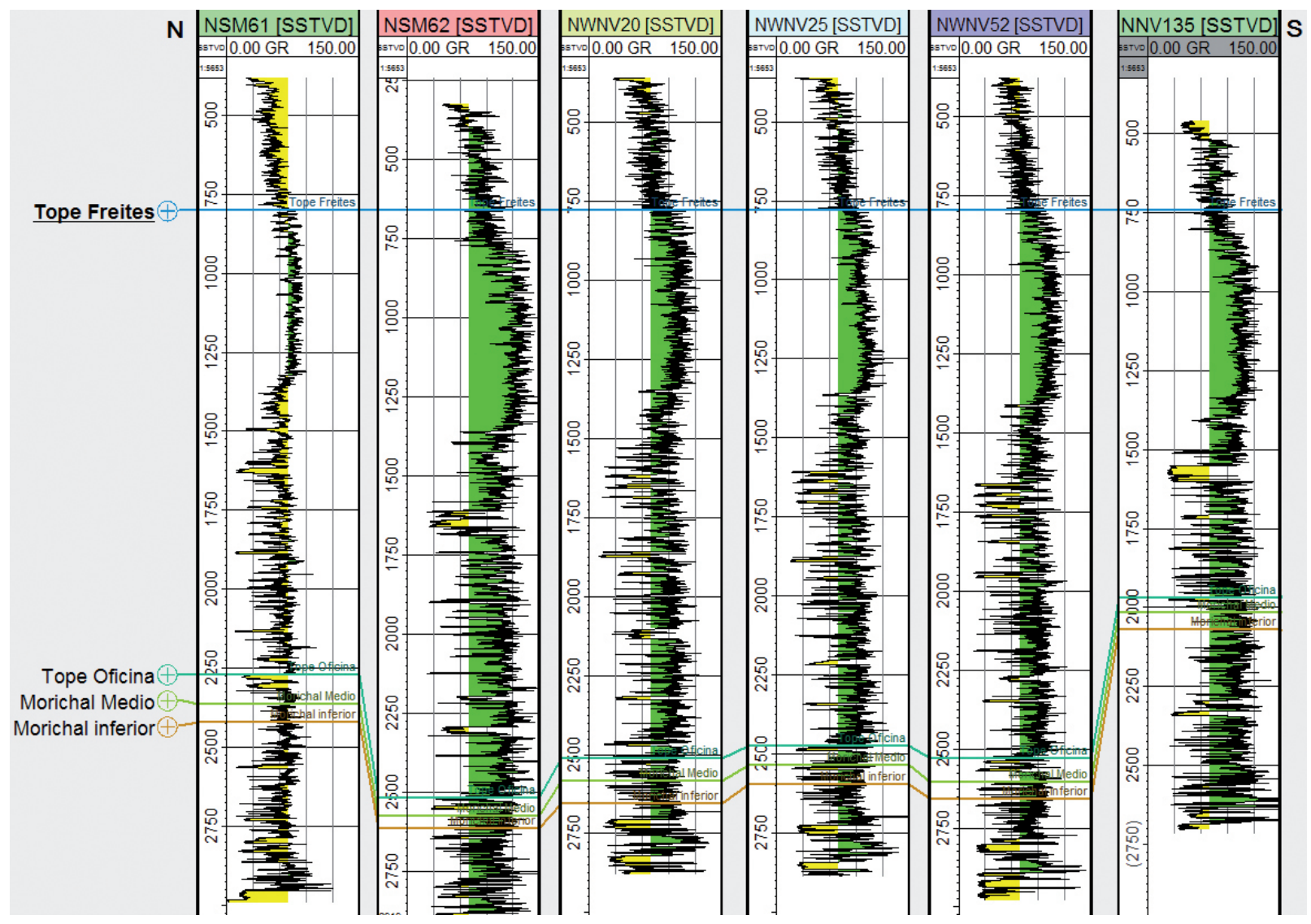

Figura13. Perfil AB N50O para topes de Formación.

Ahora bien, en cuanto al mismo perfil realizado pero para eventos estratigráficos de la zona, en la Figura 14 se observa una disminución de la granulometría de norte a sur, con tendencias más arcillosas al sur y un aumento del tamaño de grano hacia el norte, siendo representativo con la dirección de depositación de los sedimentos y el espacio de acomodamiento de la cuenca. En dicho perfil se determinaron 5 eventos transgresivos y 5 eventos regresivos, de los cuales se pudo comprobar, por la baja angularidad de los eventos observados en los registros, que las respuestas son representativos de una secuencia tipo 2 (exposición parcial de la plataforma).

Dicha exposición parcial se debe a un descenso lento del nivel eustático del mar, menor que la tasa de subsidencia, lo que representa sedimentos de plataforma sometidos a cursos de agua que originaron la variación en la granulometría observada; esto se confirma al observar las respuestas irregulares, de tipo campana y de embudo, que suponen ambientes fluvio-deltáico, que representan una típica respuesta de rayos Gamma en un registro de pozo.

En cuanto al segundo perfil (CD N60E), la distribución de los 5 eventos estratigráficos seleccionados, permitió observar las variaciones laterales para el tamaño de grano, en donde, al igual que el perfil anterior, al sur se aprecia una tendencia arcillosa, mientras que los pozos ubicados hacia el norte presentan arenas con menor contenido de arcilla. La granulometría disminuye de norte a sur con respuestas de los registros rayos gamma irregulares, en forma de campana y en embudo, los cuales son representativos de ambientes con energía de transporte cambiante, típicos de ambientes fluvio-deltaicos de abanicos fluviales y barras de desembocadura, respectivamente. Además, de acuerdo a la baja angularidad de los eventos estratigráficos observados, se corrobora la existencia de una secuencia tipo 2 en el área, lo que confirma las teorías previas de la zona en estudio (Castro, 2015). 


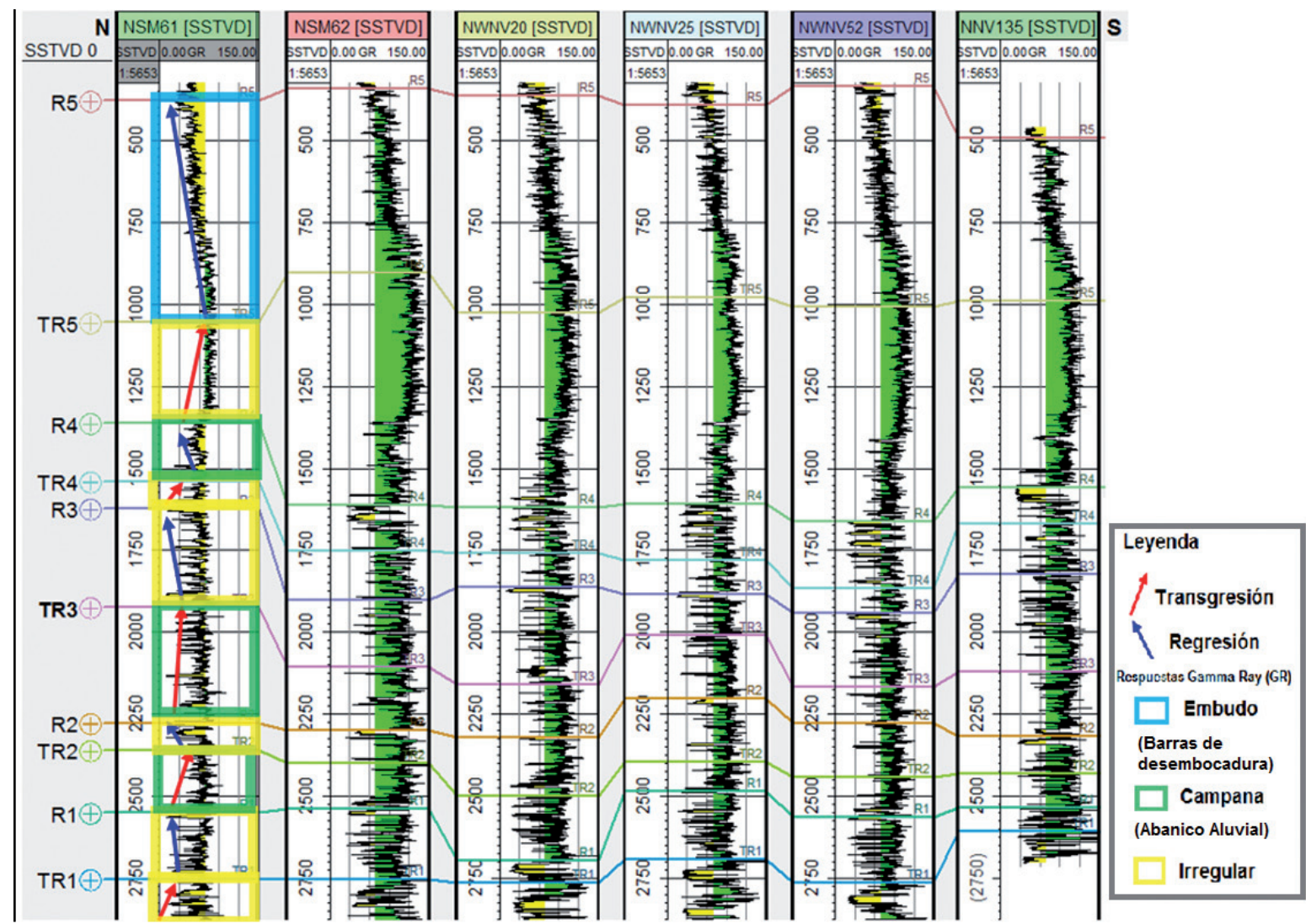

Figura 14. Perfil AB N50O para eventos estratigráficos.

Por otro lado, el mismo perfil pero para el realizado perpendicular a las fallas principales (CD N60E), en donde se representan los mismos topes estructurales, la respuesta fue similar al perfil AB N50O para los topes de Formación: de norte a sur, los topes comenzaron a profundizar, en donde se resalta un cambio brusco en profundidad de los mismos, de un pozo a otro, concordando con la presencia de la falla Tascabaña, la cual es la más representativa del área en estudio y a la disminución del espacio de acomodación hacia el delta (Quijada \& Beltrán, 2001). Además, para ambos perfiles de los topes estructurales delimitados, se observaron saltos de falla de aproximadamente 250 a 500 pies, para las principales formaciones geológicas. Esto concuerda con trabajos previos realizados en el área, en respuesta al sistema de fallas normales característico de la zona (Chramcow, 2008).

\section{RESULTADOS PARA EL CÁLCULO DE PETRÓleo ORIGINAL EN SiTIO (POES)}

El histograma de frecuencias realizado para la zona 1 (Figura 15), representa una distribución de probabilidad normal con valores de POES que oscilan entre los
4,9 MMBN y 15 MMBN aproximadamente, para los valores extremos y menos frecuentes. La media fue de 9,5 MMBN con una desviación estándar de 1,9 MMBN lo que indica un valor de casi 2 MMBN de variabilidad para los valores de POES, con valores de probabilidad de escenario negativo de $6 \mathrm{MMBN}$. Los valores con más probabilidad y mayor frecuencia de repetición, se ubican entre los 8,5MMBN (escenario medio) y los 10 MMBN, representando un escenario positivo.

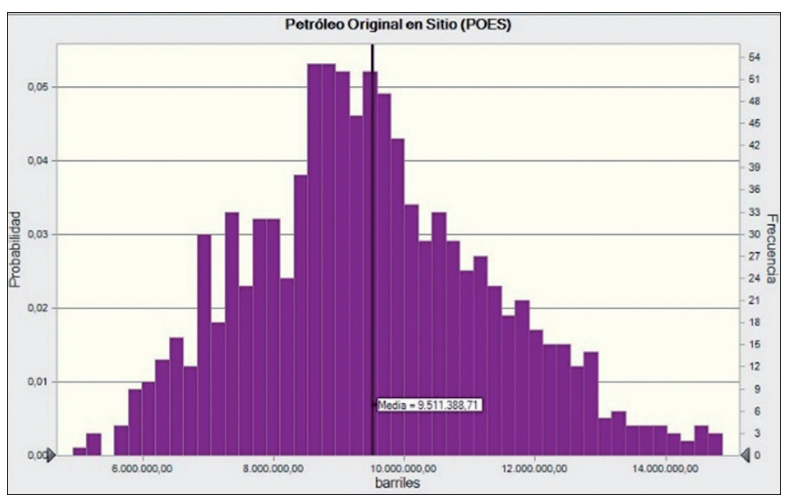

Figura 15. Histograma de frecuencias para el cálculo de Petróleo Original en Sitio (POES) para la Zona 1 
Para el histograma de frecuencias obtenido para los valores de POES en la zona 2 (Figura 16), se observa una distribución normal asimétrica con sesgo hacia los valores inferiores y una cola en el extremo derecho que representa los valores mayores del cálculo de POES. Por esta razón, debido a que la mayor concentración de los valores del histograma se relaciona con los menores valores, para el prospecto 2 se toma un escenario negativo.

Es así, como la media se ubica en $742,3 \mathrm{MBN}$ con la mayor concentración de los datos en valores de POES entre $400 \mathrm{MBN}$ y $900 \mathrm{MBN}$. Ya para esta zona, aunque se utilizó la misma área que la zona 1, el valor de POES disminuye considerablemente, lo que coincide con los espesores de arena neta petrolífera que también sufrieron un decrecimiento en los rangos de espesores para este intervalo. La desviación estándar fue de 240, $3 \mathrm{MBN}$ con valores que oscilan entre los $300 \mathrm{MMBN}$ (escenario negativo) y los 1,4 MMBN aproximadamente (escenario positivo).

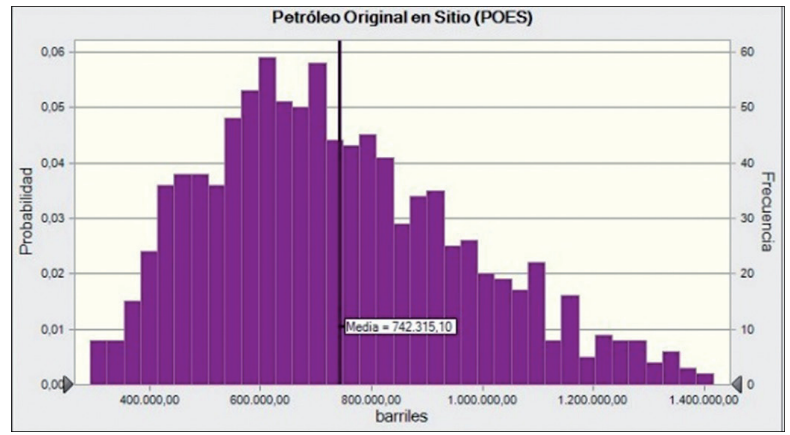

Figura 16. Histograma de frecuencias para el cálculo de Petróleo Original en Sitio (POES) para la Zona 2.

Con respecto al histograma de frecuencias para la zona 3 (Figura 17), se observa una distribución normal con valores variables que van desde los 210,8 MBN a los $898 \mathrm{MBN}$, con la mayor concentración de los datos en la zona central del gráfico en un rango de $350 \mathrm{MBN}$ a $600 \mathrm{MBN}$. Por esta razón, el prospecto 3 se ubicó en un escenario medio.

La media fue de $475 \mathrm{MBN}$ con una desviación estándar de 119,7 MBN. Obsérvese que en comparación con los histogramas anteriores, (zona 1 y 2), éste ha sido el que arrojó menores valores para el cálculo de POES, lo que coincide con los mapas de arena neta y arena neta petrolífera de la zona, que indican espesores muy pequeños para albergar hidrocarburo.

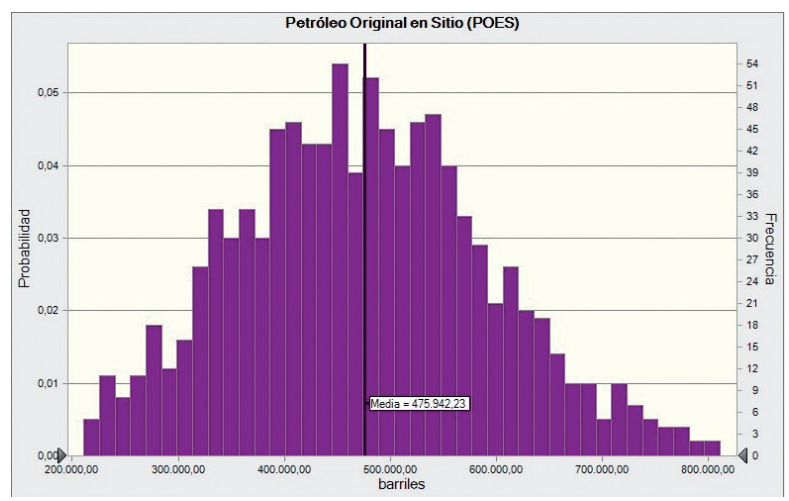

Figura 17. Histograma de frecuencias para el cálculo de Petróleo Original en Sitio (POES) para la Zona 3.

Una tabla resumen de los histogramas realizados, se muestra a continuación en base a los escenarios más probables por prospecto.

Tabla 3. Resumen de valores obtenidos para el cálculo de POES por zona.

\begin{tabular}{|cccc|}
\hline Prospecto & $\begin{array}{c}\text { Escenario } \\
\text { Negativo }\end{array}$ & $\begin{array}{c}\text { Escenario } \\
\text { Medio }\end{array}$ & $\begin{array}{c}\text { Escenario } \\
\text { Positivo }\end{array}$ \\
\hline Prospecto 1 & & $9,5 \mathrm{MMBN}$ & \\
\hline Prospecto 2 & $742,3 \mathrm{MBN}$ & \\
\hline Prospecto 3 & & $475 \mathrm{MBN}$ \\
\hline
\end{tabular}

\section{CONCLUSIONES}

Los mapas de isopropiedades generados para las 3 zonas, siguieron una tendencia a disminuir los valores de las propiedades petrofísicas analizadas, a medida que se iba profundizando en el área; es decir, los mayores valores para Arena Neta (AN), Arena Neta Petrolífera (ANP) y Porosidad $(\Phi)$, se ubicaron en los mapas generados para la Zona 1, mientras que los menores valores, se obtuvieron para las zonas inferiores, siendo coherente con el aumento del grado de arcillosidad mayor profundidad. Con base a ello, se concluye que, los mapas generados para la zona 1, se encuentran dominados por eventos estructurales, mientras que para la zona 2, estuvo dominada por eventos estratigráficos de ambientes fluvial-deltaicos, confirmando lo esperado en el área.

Los 3 prospectos propuestos, se interpretaron en base a la integración de parámetros petrofísicos y de sus mapas de isopropiedades. Dichos prospectos, mantuvieron la 
misma ubicación (en coordenadas) en todos los mapas pero a distinta profundidad, lo cual evidencia una continuidad vertical para las 3 zonas estudiadas. Por esta razón, se recomienda perforar pozos productores verticales.

De acuerdo a los perfiles realizados para los topes estructurales de Formación, se observó un comportamiento característico del área en estudio, en donde se presentan fallas normales que dan respuesta a estos cambios bruscos en los principales topes de Formación, originando un graben en el centro del mapa, aunado al aumento de espesores de sedimentos, representado en los pozos centrales de los perfiles, por las arenas con mayor contenido de arcilla a partir del tope de la Formación Freites. Cabe destacar, que esta depresión topográfica, se asemeja a estructuras tipo flor, que responden a las fallas normales características del área, también observadas en los resultados obtenidos para el levantamiento sísmicos Chimire 3D 1997 reportados por otros autores.

Con respecto a los eventos estratigráficos representados en los perfiles, se observaron transgresiones y regresiones, con respuestas en los registros de Gamma Ray irregulares, de campana y en embudo, típicos de los sistemas encadenados de bajos nivel (regresión) y alto nivel (transgresión) en plataformas clásticas que suponen ambientes fluvio-deltaico con barras de desembocaduras y abanicos fluviales, generando una secuencia tipo 2 debido a la baja angularidad de la plataforma (menor a $5^{\circ}$ ), lo cual es característico del pasado geológico de la Cuenca Oriental de Venezuela.

Finalmente, los histogramas realizados, mostraron valores decrecientes a medida que se profundizaba en el área, debido al aumento de arenas con mayor contenido de arcilla y al adelgazamiento de los espesores de los Miembros de la Formación Oficina en los estratos infraadyacentes, es por esto, que el gráfico obtenido para la zona 1 fue el que presentó mayores valores de POES mientras que el obtenido para la zona 3, representó los menores valores de espesor de arenas con hidrocarburos. Esto es coherente con lo observado en los mapas de arena neta (AN) y Arena Neta Petrolífera (ANP) en donde se propuso un buen prospecto petrolero; cabe resaltar que con el método de Monte Carlo, los valores obtenidos son variables y existe un grado de varianza para los 3 gráficos, usando un área aproximada del prospecto petrolero de 355,67 acres lo que se traduce en 1, $4 \mathrm{Km}^{2}$, además de representar un rango de espesores de arena neta petrolífera entre 120 pies a 250 pies. En consecuencia, dado el rango de variabilidad de los valores de espesor que pudieran contener el hidrocarburo deseado, se sugiere ser cauteloso en cuanto a la toma de decisiones a partir de los valores obtenidos por el cálculo de petróleo original en sitio (POES).

\section{BIBLIOGRAFÍA}

1. Acosta, E., (2006). Modelo petrofísico de yacimientos con alta saturación irreducible de agua de la formación Oficina. Trabajo especial de grado. Universidad del Zulia. Maracaibo, Venezuela.

2. Albertini, Z., \&Cadena, E., (2014). Análisis estratigráfico secuencial de la Formación Oficina ubicada en la división Carabobo de la Faja Petrolífera del Orinoco. Trabajo especial de grado. Universidad Central de Venezuela. Caracas, Venezuela.

3. Audemar, F.; I. Azpiritxaga; P. Baumann; A. Isea y M. Latreille, 1985. Marco geológico del terciario de la faja petrolífera del Orinoco, Venezuela. VI Congreso Geológico Venezolano, Caracas, 1985, S.V.G., 1: 70-108.

4. Carrero, Luis., (2013). Estudio técnico-económico para optimizar el esquema de explotación por recuperación mejorada aplicando simulación: caso yacimiento 14 ns 301, campo Chimire. Trabajo especial de grado. Universidad Central de Venezuela. Caracas, Venezuela.

5. Castro, N., (2015). Generación de modelo petrofísico-estratigráfico del campo Chimire, Estado Anzoátegui.Trabajo especial de grado. Universidad Central de Venezuela. Caracas, Venezuela.

6. Catuneanu, O., Galloway, W., Kendall, C., Miall, A., Posamentier, H., Strasser, A., and Tucker, M., (2011). Sequence Stratigraphy: Methodology and Nomenclature. Newsletters on Stratigraphy, Vol. 44/3, 173-245.

7. Chramcow, D., (2008). Interpretación sismoestratigráfica del área de Pichincha perteneciente a la Faja Petrolífera del Orinoco. Trabajo especial de grado. Universidad Simón Bolívar. Sartenejas, Venezuela.

8. Fajardo, A., \&Izarra, L., (2003). Delimitación de arenas gasíferas superiores del área Mayor de Oficina, mediante interpretación sísmica 3D, Campo Güico-Guara, Cuenca Oriental de Venezuela, Estado Anzoátegui. Trabajo especial de grado. Universidad Central de Venezuela. Caracas, Venezuela. 
9. González de Juana, C., Iturralde, J. \&Picard, X., (1980).Cuenca Petrolífera de Falcón. Geología de Venezuela y de sus Cuencas Petrolíferas. Tomo II. Caracas. Ediciones FONINVES, tomo I, Caracas.

10. Larrañaga, D., (2000). Delimitación de áreas de interés gasífero, arenas superiores de la Formación Oficina, Campos Boca y Chimire, Estado Anzoátegui. Trabajo especial de grado. Universidad Simón Bolívar. Caracas, Venezuela.

11. Hedberg, H., (1980). Guía Estratigráfica Internacional. Editorial Reverté. España.

12. Larrañaga, D., (2000). Delimitación de áreas de interés gasífero, arenas superiores de la Formación Oficina, Campos Boca y Chimire, Estado Anzoátegui. Trabajo especial de grado. Universidad Simón Bolívar. Caracas, Venezuela.

13. Martínez, H., (2007). Caracterización del modelo dinámico del yacimiento JM-19 en la arena ME-C del campo San Joaquín, en el Área Mayor de Anaco. Trabajo especial de grado. Universidad Central de Venezuela. Caracas, Venezuela.

14. Quijada, C. \& Beltrán, C., (2001). Interpretación sísmica del campo Chimire, arenas "A" y arenas "G", Formación Oficina. Informe Interno PDVSA E\&P, Venezuela.

15. Sandia, J., (2004). Interpretación sísmica del sinclinal de Morichito, al norte del Estado Monagas,
Venezuela Oriental. Trabajo especial de grado. Universidad Central de Venezuela. Caracas, Venezuela.

16. Santaniello, F., (2003). Aplicación del método de Monte Carlo en exploración y explotación de petróleo y gas natural. Trabajo especial de grado. Universidad Central de Venezuela. Caracas, Venezuela.

17. Serra, O., Baldwin, J., \&Quirein, J.A., (1980). Theory, interpretation and practicalapplications of Naural Gamma RaySpectroscopy. SPWLA, $21^{\text {th }}$ ann. Log. Symp. Trans., paper Q.

18. Serra, O., (1985). Sedimentar y environments from wire line logs. $1^{\text {st }} \& 2^{\text {nd }}$ ed., Schlumberger, M081030/ smp-7008.

19. Velásquez, L., (2003). Construcción de un volumen de velocidades interválicas para efectuar una migración $3 \mathrm{D}$ post-apilamiento en profundidad, campo Chimire-Boca, Estado Anzoátegui. Trabajo especial de grado. Universidad Central de Venezuela. Caracas, Venezuela.

20. Villalba, Y., (2011). Estimación probabilística de las reservas de hidrocarburos de los yacimientos U6 MEL 166 y U6 101 del campo Melones de la división Ayacucho, usando el método de riesgo e incertidumbre. Trabajo especial de grado. Universidad de Oriente. Puerto la Cruz, Venezuela.

Recepción: 8 de marzo de 2016

Aceptación: 2 de mayo de 2016 